(c) American Dairy Science Association, 2003.

\title{
Genetic Parameters for Body Condition Score, Body Weight, Milk Yield, and Fertility Estimated Using Random Regression Models
}

\author{
D. P. Berry, ${ }^{\star} \dagger$ F. Buckley, ${ }^{\star}$ P. Dillon, ${ }^{\star}$ R. D. Evans, ${ }^{\star}$ \\ M. Rath, $\dagger$ and R. F. Veerkampł \\ *Dairy Production Department, Teagasc, \\ Moorepark Production Research Centre, Fermoy, Co. Cork, Ireland \\ †Department of Animal Science, Faculty of Agriculture, \\ University College Dublin, Belfield, Dublin 4, Ireland \\ ¥ Animal Resources Development, Animal Sciences Group, \\ Wageningen, 8200 AB Lelystad, The Netherlands
}

\section{ABSTRACT}

Genetic (co)variances between body condition score (BCS), body weight (BW), milk yield, and fertility were estimated using a random regression animal model extended to multivariate analysis. The data analyzed included 81,313 BCS observations, 91,937 BW observations, and 100,458 milk test-day yields from 8725 multiparous Holstein-Friesian cows. A cubic random regression was sufficient to model the changing genetic variances for BCS, BW, and milk across different days in milk. The genetic correlations between BCS and fertility changed little over the lactation; genetic correlations between BCS and interval to first service and between BCS and pregnancy rate to first service varied from -0.47 to -0.31 , and from 0.15 to 0.38 , respectively. This suggests that maximum genetic gain in fertility from indirect selection on BCS should be based on measurements taken in midlactation when the genetic variance for BCS is largest. Selection for increased BW resulted in shorter intervals to first service, but more services and poorer pregnancy rates; genetic correlations between $\mathrm{BW}$ and pregnancy rate to first service varied from -0.52 to -0.45 . Genetic selection for higher lactation milk yield alone through selection on increased milk yield in early lactation is likely to have a more deleterious effect on genetic merit for fertility than selection on higher milk yield in late lactation.

(Key words: body weight, body condition score, fertility, random regression)

Abbreviation key: DairyMIS = dairy management information system, IFS = interval to first service,

Received May 8, 2003.

Accepted June 30, 2003.

Corresponding Author: D. P. Berry; e-mail: dberry@moorepark. teagasc.ie.
$\mathbf{L 0}=$ random regression model with only the linear intercept term included, $\mathbf{L 1}$ = linear random regression model, $\mathbf{L 2}$ = quadratic random regression model, L3 $=$ cubic random regression model, NAHF $=$ North American Holstein Friesian, NS = number of services per cow, PR63 = pregnant $63 \mathrm{~d}$ after the start of the breeding season, PRFS = pregnancy rate to first service, $\mathbf{R R M}=$ random regression model.

\section{INTRODUCTION}

Fertility in dairy cattle is strongly influenced by both the extent and duration of negative energy balance (Butler et al., 1981; Butler and Smith, 1989). However, energy balance is difficult to measure in large populations, leading to increased interest in other traits (BCS, BW, and milk yield), which may be indicators of energy balance (Coffey et al., 2001) and may be subsequently related to the health and fertility status of an animal. However, the genetic correlation between an indicator trait and fertility may differ depending on the stage of lactation when the indicator trait is measured. This hypothesis can be tested by treating indicator traits measured at different stages of lactation as separate, yet not mutually exclusive traits. Multivariate analyses and covariance functions provide a method of treating longitudinal traits measured at different stages of lactation as separate traits with a correlation structure between them.

Previous studies using multivariate analyses suggest favorable genetic correlations between fertility with BCS (Dechow et al., 2001; Pryce et al., 2001; Royal et al., 2002; Berry et al., 2003) and antagonistic genetic correlations between fertility and milk yield (Hoekstra et al., 1994; Grosshans et al., 1997; Royal et al., 2000; Pryce et al., 2001; Roxström et al., 2001; Evans et al., 2002). Pryce et al. (2001) reported negative genetic correlations between average BCS and calving interval $(-0.36)$, days to first heat $(-0.41)$, and 
days to first service (-0.54). Veerkamp et al. (2000) reported that animals with a high genetic merit for BW tended to commence luteal activity earlier. Genetic correlations estimated between $\mathrm{BW}$ and other fertility measures suggested that genetically heavier cows had poorer pregnancy rates and required more services (Berry et al., 2003). Many of the above studies (Pryce et al., 2000; Veerkamp et al., 2000; Dechow et al., 2001; Berry et al., 2003) showed correlations of varying strength depending on when during lactation the indicator trait was measured, although in most cases the direction of the correlations remained relatively constant.

Covariance functions provide alternatives to multivariate analyses and may be considered as infinitedimensional equivalents to covariance matrices for a given number of traits across a continuous scale (e.g., lactation). Meyer (1998) showed that covariance functions could readily be estimated with a random regression model incorporating orthogonal polynomials. Random regression models have previously been applied to describe the (co)variances of BCS (Koenen and Veerkamp, 1998; Jones et al., 1999; Veerkamp et al., 2001), BW (Koenen and Veerkamp, 1998; Veerkamp and Thompson, 1999), DMI (Koenen and Veerkamp, 1998; Veerkamp and Thompson, 1999), and milk yield (Jamrozik and Schaeffer, 1997; Rekaya et al., 1999) over a lactation. However, very few studies are available (Veerkamp et al., 2001) that estimate the genetic correlations between a longitudinal trait (i.e., BCS, $\mathrm{BW}$, and milk) and fertility using a random regression model extended to a multivariate analysis. The dataset used in the present study is relatively unique in that it contains a large number of animals with a relatively large number of BCS and BW records per animal across $2 \mathrm{yr}$. Hence, this dataset is particularly suited to the estimation of correlations between fertility with BCS, BW, and milk yield treated as longitudinal traits.

The objectives of the present study were to estimate using random regression models the (co)variances of BCS, BW, and milk yield over a lactation, and subsequently to estimate the genetic correlations between each of the three longitudinal traits with fertility by extending the random regression model to a multivariate analysis. This will provide an indication of how the genetic correlations between each of the longitudinal traits and fertility may differ depending on the stage of lactation when the longitudinal trait is measured.

\section{MATERIALS AND METHODS}

This study, carried out over 2 yr (1999 and 2000), was composed of 78 spring-calving dairy herds (74
Table 1. Number of records for BCS, BW, and milk yield at different days of lactation (DIM).

\begin{tabular}{lrrr}
\hline DIM/Trait & \multicolumn{1}{l}{ BCS } & \multicolumn{1}{c}{ BW } & \multicolumn{1}{c}{ Milk } \\
\hline $1-50$ & 23,161 & 25,745 & 13,195 \\
$51-100$ & 18,569 & 21,207 & 20,126 \\
$101-150$ & 13,674 & 16,579 & 20,262 \\
$151-200$ & 9760 & 12,844 & 19,524 \\
$201-250$ & 4441 & 6802 & 17,807 \\
$251-300$ & 4789 & 3724 & 9544 \\
$301-350$ & 6919 & 5036 & \\
Total & 81,313 & 91,937 & 100,458 \\
\hline
\end{tabular}

commercial and 4 research herds) in the south of Ireland with a potential 8928 spring-calving cows available for inclusion in the dataset. Herd size ranged from 30 to 240 cows. All herds were incorporated into the dairy management information system (DairyMIS) run by Moorepark (Crosse, 1986). The DairyMIS is a recorder-based computerized system collecting detailed stock, farm inputs, production, and reproduction information on a monthly basis.

\section{Animal Traits}

A total of 8499 cows from 76 herds had recorded spring calving dates and one or more BCS (scale 1 to 5) or BW records, whereas 8767 cows from 78 herds had recorded spring calving dates and one or more milk records. Berry et al. (2002) explains the recording of each trait in more detail. Trained Teagasc personnel visited the farms up to 9 times per year. Visits were carried out at two and a half to four weekly intervals, with visits being more frequent in early lactation. During visits, all cows in the herd were recorded for $\mathrm{BW}$ and BCS. Only records between calving and 350-d postpartum were included in the analysis for BCS and BW. Because the average lactation length in Ireland is around $270 \mathrm{~d}$ (Irish cattle breeding statistics, 2002), there were only 885 milk test-day records after $300 \mathrm{~d}$ of lactation. It is for this reason that only records between calving and 300-d postpartum were included in the analysis for milk production. The total number of test-day records included in the analysis were: 81,313 for BCS, 91,937 for BW, and 100,458 for milk yield (Table 1).

Four fertility variables similar to those used internationally (Grosshans et al., 1997; Veerkamp et al., 2001; Evans et al., 2002) were calculated: interval to first service (IFS), pregnancy rate to first service (PRFS), number of services per cow (NS), and pregnant $63 \mathrm{~d}$ after the start of the breeding season (PR63). The start of the breeding season for each herd was defined as the first service date recorded in that herd; start of breeding dates were available for both 
years of the study. Pregnant $63 \mathrm{~d}$ after the start of breeding was chosen to identify cows that conceived relatively early in the breeding season, an attribute conducive to a successful compact spring calving system. We identified first service records for a total of 8315 cows. In Ireland, most farmers use artificial insemination for the first $6 \mathrm{wk}$ of the breeding season and natural mating thereafter. During the breeding season, $92 \%$ of farmers observed cows more than twice daily for estrus, whereas $99 \%$ of farmers used tail paint and/or a vasectomized bull as an aid to estrous detection. This facilitated accurate recording of all services. Beginning 40 to $50 \mathrm{~d}$ after the start of the breeding season, all herds were visited on three or four occasions, at approximately 40-d intervals, to perform pregnancy diagnosis by transrectal ultrasound imaging (Aloka 210D* II, 7.5 MH3, Aloka Co. Ltd, Tokyo, Japan). Cows inseminated for at least $28 \mathrm{~d}$ and subsequently not observed in estrus were scanned to confirm pregnancy. Subsequently, all cows in the study were determined to be pregnant or not by rectal palpation of the entire herd at least $56 \mathrm{~d}$ after the end of the defined breeding season. Hence, all cows were serviced at least $56 \mathrm{~d}$. No pregnancy diagnosis was carried out thereafter as part of the study. Further losses beyond this point were not considered.

The proportion of North American HolsteinFriesian (NAHF) genetics for each sire/maternal grand sire present in the dataset was calculated as outlined by Berry et al. (2002). The percentage of NAHF genes in the cows within the present study varied from 0 to $75 \%$; average NAHF percentage was $51 \%$. Berry et al. (2002) explains the pedigree information for the animals in the study in more detail. The additive genetic relationship matrix consisted of 20,699 animals.

\section{Data Analysis}

(Co)variances within traits. Genetic (co)variances within each of the three longitudinal traits (BCS, BW, and milk yield) were estimated using ASREML (Gilmour et al., 2003). The models used were progressively built up from a univariate analysis treating the longitudinal trait at different stages of lactation as separate traits, to a multivariate analysis and subsequently a random regression model. However, the univariate analysis within different lactation stages compared poorly with the random regression model analysis, and therefore only multivariate analyses were subsequently performed.

The following linear animal model was used for the multivariate analyses within traits:

$$
\begin{gathered}
Y_{i j k m p}=H_{Y T D_{j}}+\sum_{i=1}^{2} b_{i} \text { Hol }^{i}+l_{k} \times\left(\sum_{l=1}^{10} b_{l} \text { DIM }^{l}\right)+l_{k} \\
\times\left(\sum_{m=1}^{10} b_{m} \text { CON }^{m}\right)+a_{i}+\text { PEwithin }_{i}+\text { PEbetween }_{i} \\
+e_{i j k m p},
\end{gathered}
$$

where:

$$
\begin{aligned}
& Y_{i j k m p}=\text { observation for trait } p \text { on ani- } \\
& \text { mal } i \\
& H Y T D_{j}=\text { fixed effect of herd by year by } \\
& \text { test-day }(j=3168) \text {, } \\
& \sum_{i=1}^{2} b_{i} \mathrm{Hol}^{i}=\begin{array}{l}
\text { fixed effect of a quadratic poly- } \\
\text { nomial regression on the per- }
\end{array} \\
& \text { centage of North American } \\
& \text { Holstein-Friesian genes in ani- } \\
& \text { mal } i \text {, } \\
& l_{k} \times\left(\sum_{l=1}^{10} b_{l} D I M^{l}\right)=\begin{array}{l}
\text { Fixed effect of parity by days } \\
\text { in milk interaction }(k=1,2,3, \\
4+),
\end{array} \\
& l_{k} \times\left(\sum_{m=1}^{10} b_{m} C^{m}\right)=\begin{array}{l}
\text { Fixed effect of parity by days } \\
\text { conceived interaction }(k=1,2, \\
3,4+),
\end{array} \\
& a_{i}=\text { random effect for an additive } \\
& \text { genetic effect, } \\
& P E \text { within }_{i}=\text { random effect for the within- } \\
& \text { year permanent environment } \\
& \text { of each cow, }
\end{aligned}
$$

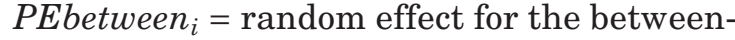

$$
\begin{aligned}
& \text { year permanent environment } \\
& \text { of each cow, and } \\
& e_{i j k m p}=\text { random residual term } \text {. }
\end{aligned}
$$

The parity $\times$ DIM interaction included in the model accounted for the significantly $(P<0.001)$ different lactation curves for BCS, BW, and milk yield in the different parities. Similarly, the parity $\times$ days conceived interaction accounted for the significantly $(P<$ 0.001 ) different effects of conception on BCS, BW, and milk yield in different parities.

Prior to the multivariate analyses for BCS and BW, the interval from calving to $350-\mathrm{d}$ postpartum was divided into seven blocks, each $50 \mathrm{~d}$ in length; for milk yield, calving to 300-d postpartum was divided into six blocks. Only records observed within the 50-d time frame set for each block were considered for inclusion in that block. Animals with no records within a block had their value treated as missing. This analysis assumed homogenous residual variances within block but heterogeneous residual variances between blocks. The number of records per block for BCS, BW, and milk yield are summarized in Table 1 . 
A $7 \times 7$ multivariate analysis for each trait resulted in some parameters becoming bound at the ends of the parameter space. It is for this reason that two separate multivariate analyses were performed: all even groups together in one analysis $(3 \times 3)$ and all odd groups together in another analysis (a $4 \times 4$ for $\mathrm{BCS}$ and BW and a $3 \times 3$ for milk yield). Residual covariances between blocks were not estimated and were assumed to be zero.

The random regression model applied to the same data was similar to the model above except that the additive genetic component and permanent environmental component within years were both modeled using a set of orthogonal polynomials across DIM. The order of polynomial fitted was consistently the same for both components. Similar to the multivariate analyses, the residual variances were estimated within the seven blocks (six blocks for milk yield), each $50 \mathrm{~d}$ in length, from d 0 to 350 of lactation (d 0 to 300 for milk yield). Residual covariances between blocks were assumed to be zero and are therefore likely to have entered the additive genetic and permanent environmental components.

Parsimonious covariance function. A covariance function with a lower-order polynomial (reduced fit) for the additive genetic component or the permanent environmental component may be consistent with a full-order covariance function (equivalent to a multivariate analysis with each DIM treated as a separate trait), but require fewer parameters to be estimated. The criteria set down to obtain the most parsimonious covariance function were: 1) the likelihood test of two nested models were compared using the test-statistic $=-2 \times$ difference in the log-likelihood between nested models assuming a $\chi^{2}$ distribution with $m$ degrees of freedom, where $m$ is the difference in the number of estimated covariance parameters between the nested models (Wilks, 1938); 2) the eigenvalues of the additive genetic and permanent environmental covariance matrix were calculated to determine the contribution of each extra term to the overall variation in the curve; 3 ) the estimated variances from the random regression model were visually compared to the multivariate analyses; and 4) the residual variances estimated for each order were compared against each other.

(Co)variances between traits. Each multivariate and random regression model analysis within the longitudinal traits themselves was further extended to include a fertility trait measured once a year; this allowed the estimation of the genetic correlations between each of the longitudinal traits with each of the fertility traits. Herd-year-season groups were formed. Season was defined as month of calving. Herd-year- season groups with less than four cows had their records moved into an adjoining season group from the same herd to facilitate a more accurate estimate. The fertility trait was adjusted for herd-year-season of calving and also a quadratic regression on calving to the start of the breeding season and a quadratic regression on calving to the end of the breeding season as in Berry et al. (2003). Furthermore, the regressions on DIM and days conceived, as well as the herd-yeartest-day interaction, were only applied to the longitudinal trait.

It was only possible to estimate the between-year permanent environmental variance for the fertility trait while both the within-year and the between-year permanent environmental variance was estimable for the longitudinal trait in the multivariate and random regression model analyses. To overcome this, the residual variance of the fertility trait was set to zero, thus the residual variance of the fertility trait entered the within-year permanent environmental component; this facilitated a within-year permanent environmental correlation structure between the fertility trait and the longitudinal trait. Between-years permanent environmental (co)variances were also estimated.

Extending the random regression model to a multivariate analysis facilitated the estimation of a new covariance matrix, which included the variance of the fertility trait, the covariance function of the longitudinal trait, and the covariances between the fertility trait and the covariance function. This new covariance matrix was fitted to both the additive genetic component and to the permanent environmental component within-year. The orders of fit of the additive genetic and permanent environmental effects were determined from the univariate random regression model. Residual variances were again estimated within each of the seven blocks for BCS and BW and within six blocks for milk yield. The genetic correlations between the covariance function and the fertility traits were calculated as described by Veerkamp et al. (2001).

\section{RESULTS}

Table 2 presents the log-likelihoods and the eigenvalues of the additive genetic covariance function obtained for the different order of fits for each of the three longitudinal traits. Each increase in order fitted the data significantly $(P<0.001)$ better than the previous order. The intercept term accounted for over $68 \%$ of the additive genetic variation for both BCS and BW but tended to be slightly less important for milk yield, where it accounted for over $57 \%$ of the additive genetic 
Table 2. Log-likelihoods and proportion of the additive genetic covariance function attributed to each eigenvalue for the first four orders of BCS, BW, and milk yield.

\begin{tabular}{|c|c|c|c|c|c|c|}
\hline Trait $^{1}$ & Order & Log-L & L0 & L1 & L2 & L3 \\
\hline \multicolumn{7}{|l|}{$\mathrm{BCS}$} \\
\hline & L0 & $-287,948$ & 1.000 & & & \\
\hline & L1 & $-285,495$ & 0.970 & 0.030 & & \\
\hline & L2 & $-283,501$ & 0.711 & 0.276 & 0.013 & \\
\hline & L3 & $-283,184$ & 0.683 & 0.237 & 0.078 & 0.002 \\
\hline \multicolumn{7}{|l|}{ BW } \\
\hline & L0 & $-328,460$ & 1.000 & & & \\
\hline & $\mathrm{L} 1$ & $-324,044$ & 0.959 & 0.041 & & \\
\hline & L2 & $-321,805$ & 0.828 & 0.159 & 0.013 & \\
\hline & L3 & $-320,958$ & 0.831 & 0.119 & 0.048 & 0.002 \\
\hline \multicolumn{7}{|l|}{ Milk } \\
\hline & L0 & $-148,327$ & 1.000 & & & \\
\hline & L1 & $-144,150$ & 0.735 & 0.265 & & \\
\hline & L2 & $-143,077$ & 0.640 & 0.246 & 0.114 & \\
\hline & L3 & $-142,631$ & 0.573 & 0.221 & 0.124 & 0.082 \\
\hline
\end{tabular}

${ }^{1} \mathrm{BCS}=$ Body condition score; BW $=$ Body weight; L0 = Random regression model with only the linear intercept term included; L1 = Linear random regression model; L2 = Quadratic random regression model; L3 = Cubic random regression model.

variation. The cubic term of the random regression model for BCS, BW, and milk yield accounted for 0.2 , 0.2 , and $8.2 \%$ of the additive genetic variation, respectively.

The reduction in residual variances as a consequence of an increase in the order of the random regression model tended to decline as the order of the random regression increased and on average was reduced by 5,8 , and $6 \%$ when the order was increased from a quadratic random regression model (L2) to cu- bic random regression model (L3) for BCS, BW, and milk yield, respectively.

\section{Genetic Variances}

Figures 1, 2, and 3 graphically illustrate the additive genetic variances estimated using the multivariate and random regression model for BCS, BW, and milk yield, respectively. The genetic variances estimated with the random regression model were gener-

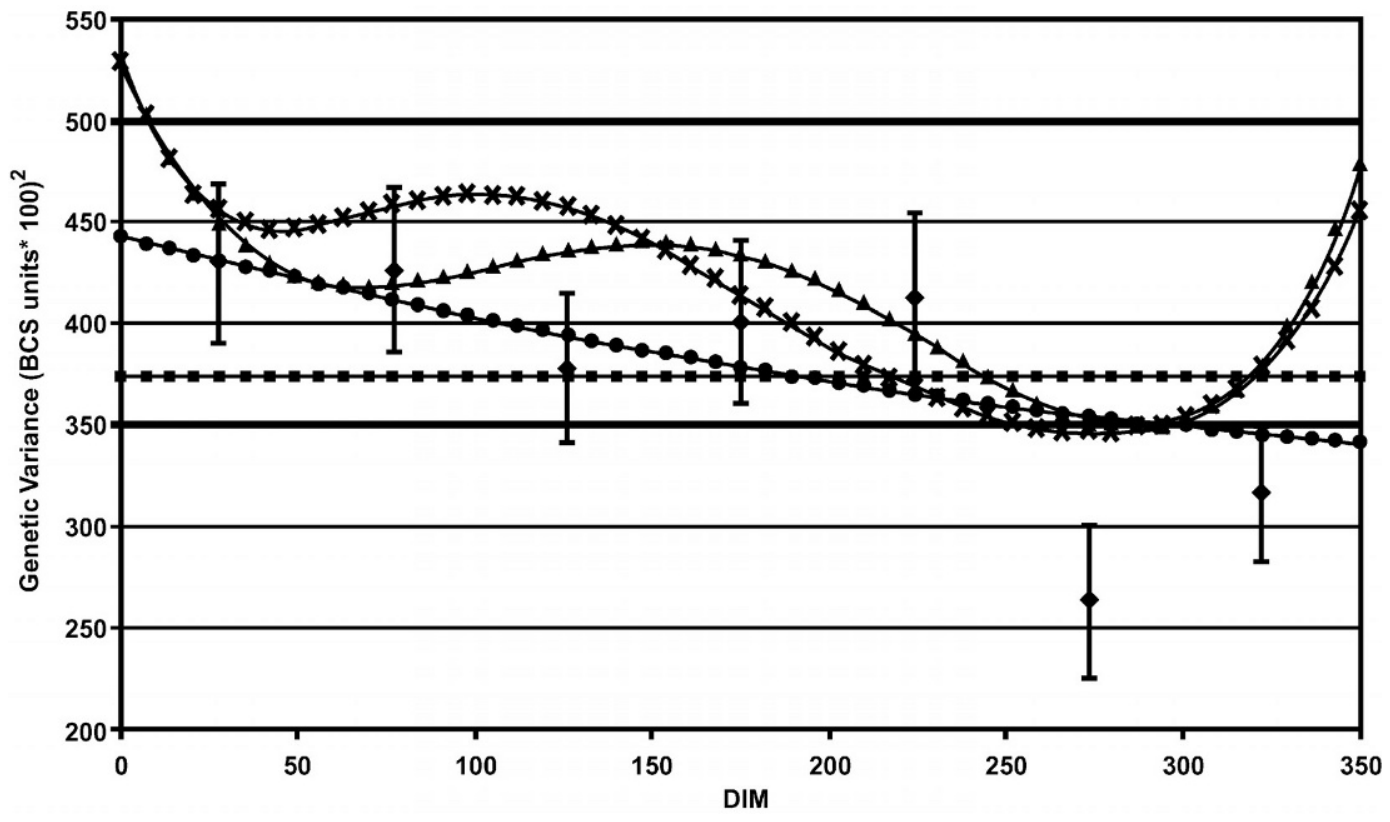

Figure 1. Additive genetic variance for body condition score at different days in milk (DIM) using a multivariate analysis $(\bullet \pm 1$ SE) and the L0 $(\mathbf{\square})$, L1 $(\bullet)$, L2 $(\mathbf{\Delta})$, and L3 $(\mathbf{x})$ random regression models. 


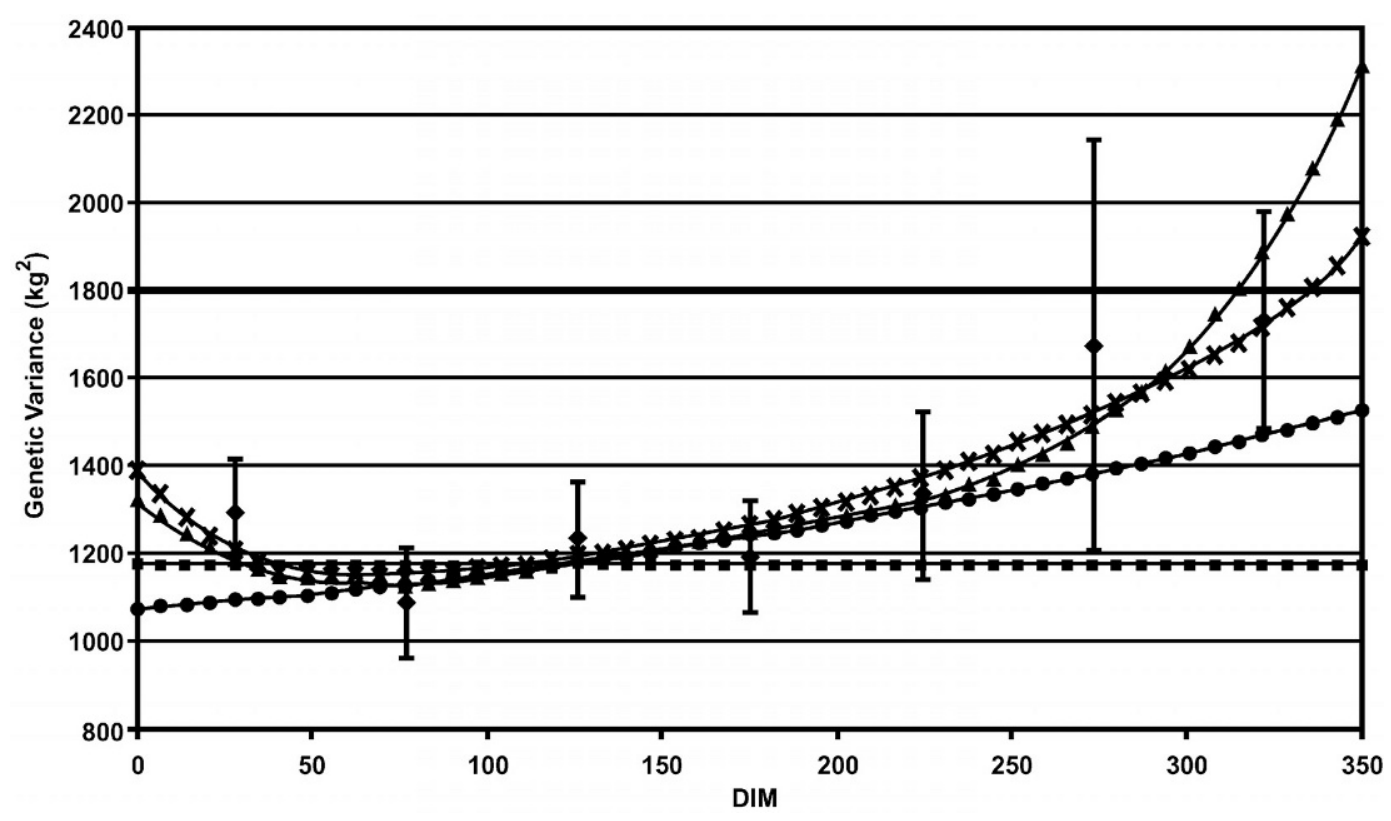

Figure 2. Additive genetic variance for body weight at different DIM using a multivariate analysis $(\bullet \pm 1 \mathrm{SE})$ and the L0 (匹), L1 (•), L2 $(\boldsymbol{\Lambda})$, and L3 $(\mathbf{x})$ random regression models.

ally larger than those estimated with the multivariate analyses.

The estimated genetic variance for BCS over the lactation ranged from $263 \mathrm{BCS}$ units $(\times 100)^{2}$ to 450 BCS units $(\times 100)^{2}$ using the multivariate model, whereas those estimated with the L3 random regression model varied from 346 to 530 BCS units $(\times 100)^{2}$ (Figure 1). Heritability estimates for BCS at different stages of lactation were smallest at d 0 of lactation (0.39) and largest at d 105 of lactation (0.51).

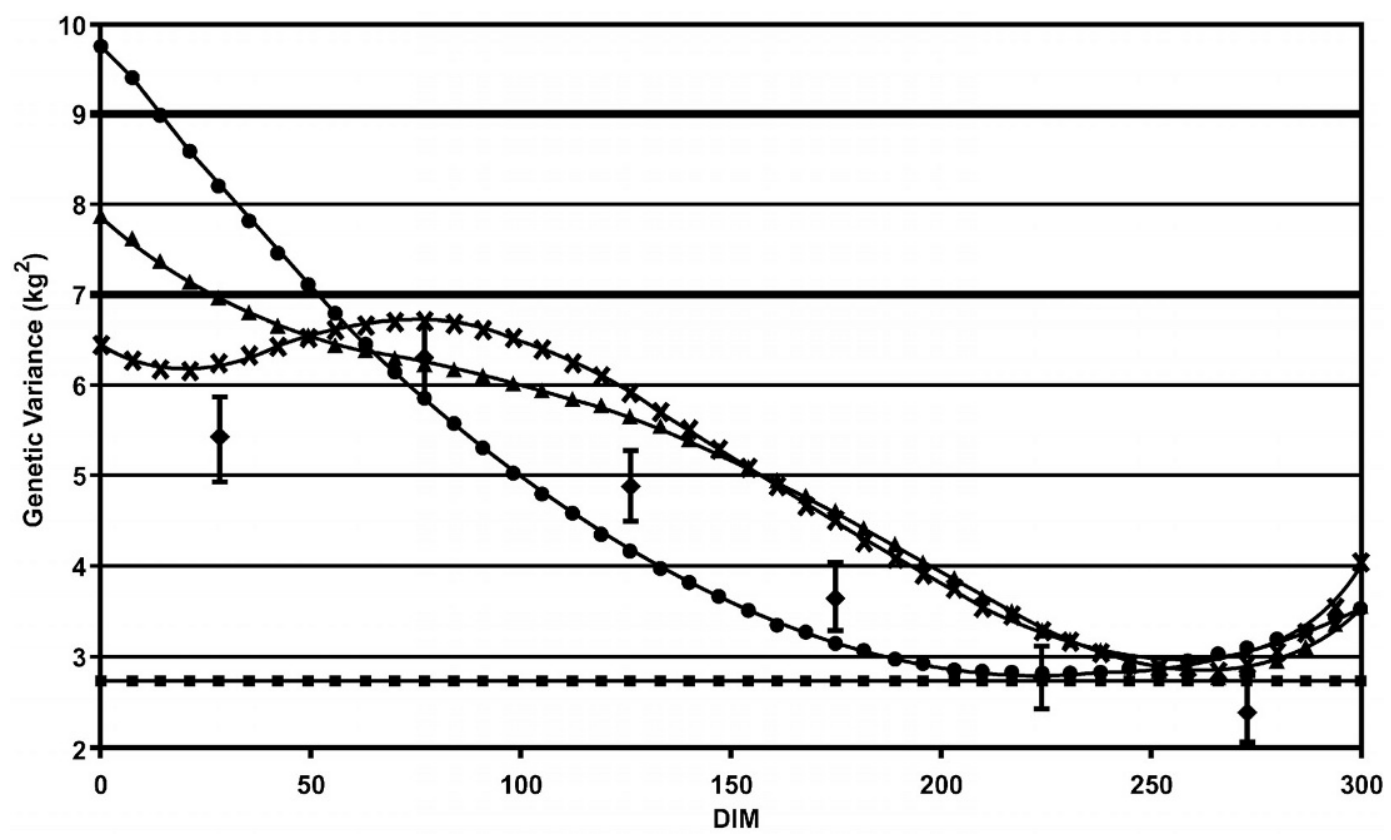

Figure 3. Additive genetic variance for milk test-day yield at different DIM using a multivariate analysis ( $\pm 1 \mathrm{SE})$ and the L0 ( $\square$ ),

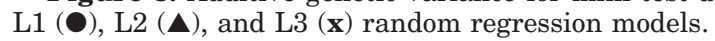


Figure 2 illustrates a continuous increase in genetic variance for $\mathrm{BW}$ from d 63 of lactation as estimated with the L3 random regression model. Heritability estimates for BW ranged from 0.48 ( $d 0$ of lactation) to 0.61 (d 294 of lactation) when using the L3 random regression model.

The genetic variances for milk yield estimated using the L3 random regression model varied from 2.8 to $6.7 \mathrm{~kg}^{2}$ (Figure 3); heritability estimates varied from 0.26 at $\mathrm{d} 0$ of lactation to 0.44 at d 105 of lactation. Both the genetic variance and the heritability for milk yield tended to be largest in early lactation.

\section{Genetic Correlations}

Genetic correlations between BCS at calving and BCS later in lactation decreased to 0.67 at $\mathrm{d} 147$ of lactation and increased thereafter. Genetic correlations between BW at calving and BW in later lactation decreased to 0.85 at $\mathrm{d} 140$ of lactation and increased thereafter. Genetic correlations between milk yield at different stages of lactation continually declined as the interval between test days increased; the lowest genetic correlation (0.07) was between milk test-day yield directly postpartum and milk test-day yield at d 300 of lactation.

Figure 4 shows very close agreement between the random regression model and the multivariate models in the estimation of genetic correlations among BCS with IFS, NS, and PR63. All genetic correlations estimated with the L3 random regression model were within one standard error of the genetic correlations estimated with the multivariate model at the same DIM. Genetic correlations estimated with the L3 random regression model between BCS with IFS ranged from -0.47 to -0.31 and between BCS with NS ranged from -0.34 to -0.17 . Genetic correlations estimated with the L3 random regression model between BCS and PR63 ranged from 0.15 to 0.40 , while the genetic correlations between BCS and PRFS ranged from 0.15 to 0.38 (results not shown). There was a tendency for BCS in mid to late lactation to be more strongly correlated with improved fertility than BCS in early lactation across both the random regression model and the multivariate analyses.

The genetic correlations between BW and IFS were all negative, between BW and NS were all positive, and between BW and PR63 were all negative across both the random regression model and multivariate models fitted (Figure 5). The magnitude of the correlation estimated with the L3 random regression model ranged from -0.69 to -0.51 between BW with IFS and from 0.34 to 0.38 between BW and NS. Genetic correlations estimated with the L3 random regression model between BW and PR63 varied from -0.22 to -0.12 , whereas correlations between BW and PRFS ranged from -0.52 to -0.45 (results not shown). Therefore although heavier animals tend to be served sooner, they have poorer overall pregnancy rates $63 \mathrm{~d}$ after the start of the breeding season, which may be in part due to their lower PRFS.

Genetic correlations between milk yield and fertility (Figure 6) were generally antagonistic with the exception of milk test-day yield in early lactation with PR63 and IFS, which were both near zero. All genetic correlations estimated with the random regression model were within one standard error of the genetic correlations estimated with the multivariate model at the same DIM. The magnitude of the correlation estimated with the L3 random regression model ranged from 0.08 to 0.35 between milk yield with IFS and from 0.28 to 0.58 between milk yield and NS. Genetic correlations estimated with the L3 random regression model between milk yield and PR63 varied from -0.30 to 0.09 ; the correlations were all negative after $d 7$ of lactation. Genetic correlations between milk yield and PRFS ranged from -0.70 to -0.24 (results not shown).

\section{DISCUSSION}

The objectives of this study were to estimate, using random regression models, the genetic (co)variances of three longitudinal traits: BCS, BW, and milk yield at different stages of lactation and subsequently to estimate the genetic correlations between each of the longitudinal traits and fertility. Indications were that a third- order random regression model was most appropriate in modeling the change in genetic variance over a lactation for BCS, BW, and milk yield. Genetic correlations between BCS at different stages of lactation with IFS, NS, PRFS, and PR63 were consistently favorable although the magnitude of the correlations changed slightly over the lactation. Increases in BW were associated with reduced IFS, increased NS, reduced PRFS, and reduced PR63. Genetic correlations involving milk test-day yield and fertility were generally antagonistic.

\section{Parsimonious Covariance Function}

Methods of choosing the most parsimonious covariance function for a dataset have been somewhat ambiguous in the literature. The likelihood ratio test has been adopted in some studies (Kirkpatrick et al., 1990) with its associated disadvantages outlined by Stram and Lee (1994). Most studies, however, combine other tests in association with the likelihood ratio test; Koenen and Veerkamp (1998) used the Akaikes Information Criterion (Akaike, 1973), whereas Veerkamp et al. (2001) 
(a)

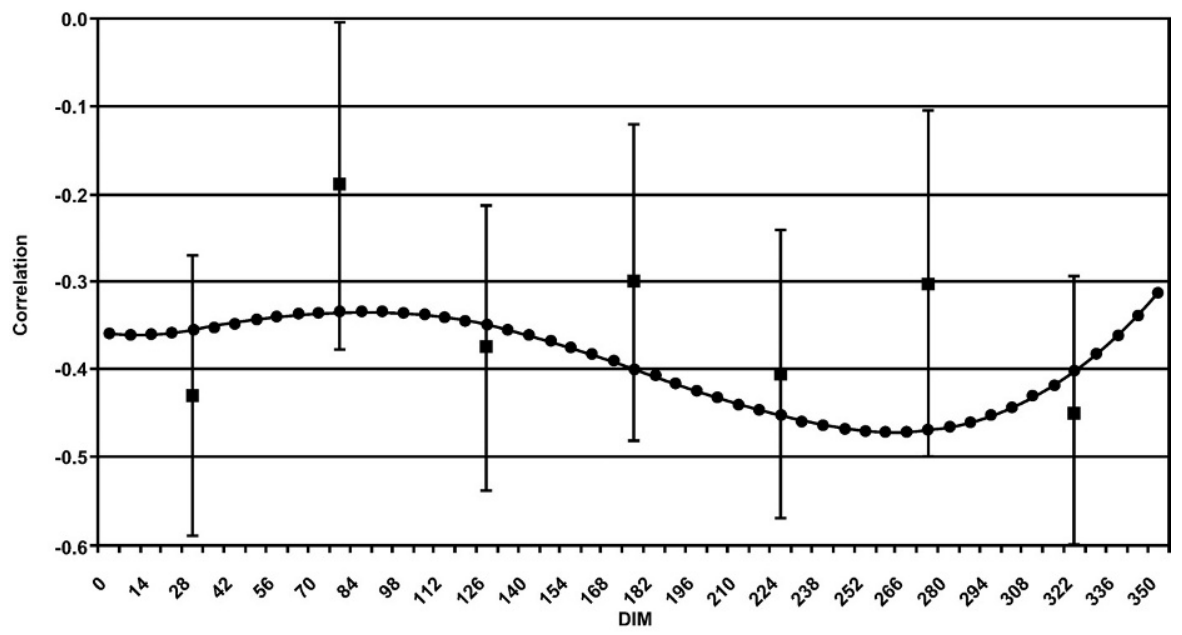

(b)

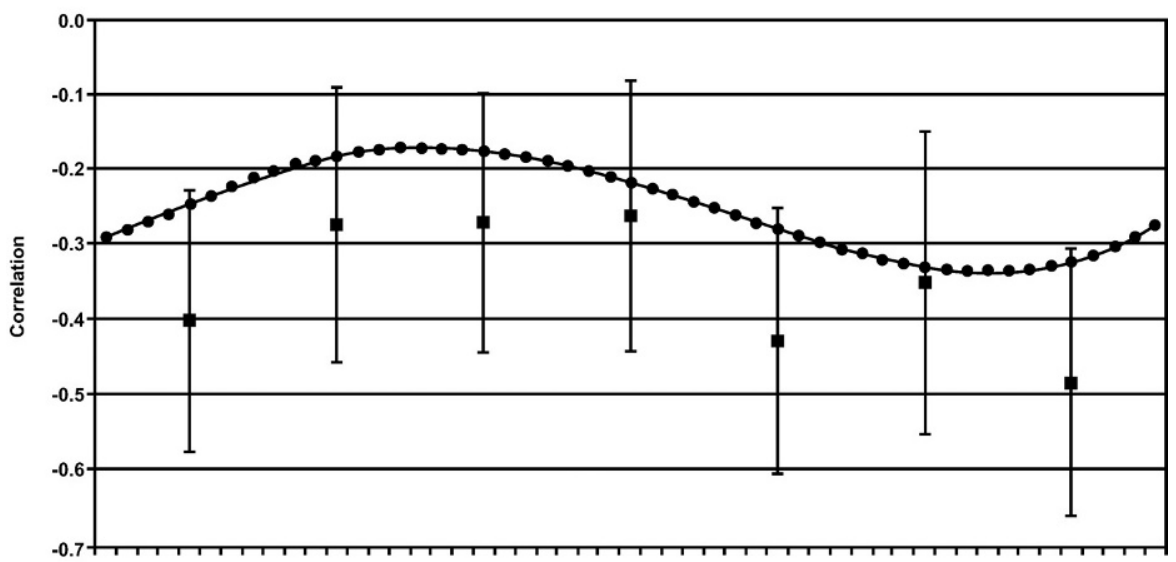

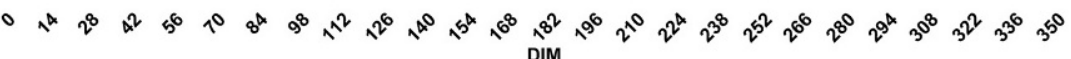

(c)

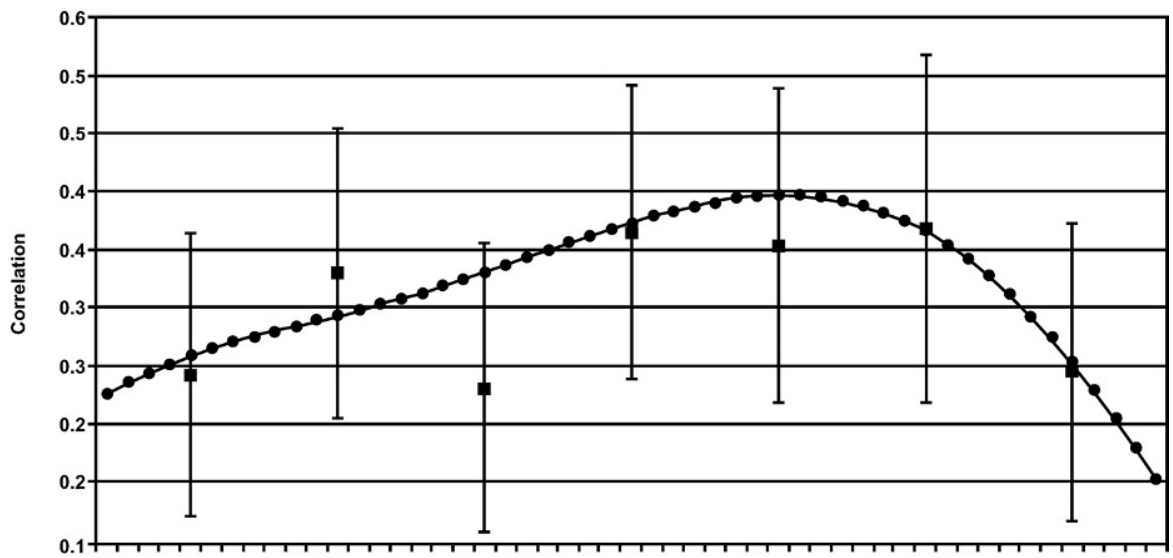

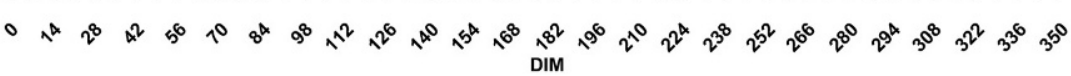

Figure 4. Genetic correlations between BCS at different stages of lactation (DIM) with (a) interval to first service ( random regression model; multivariate); (b) number of services ( random regression model; multivariate); and (c) pregnant $63 \mathrm{~d}$ after the start of breeding ( random regression model; multivariate). Also included in the graph are the SE of the correlations for the multivariate analysis. 
(a)

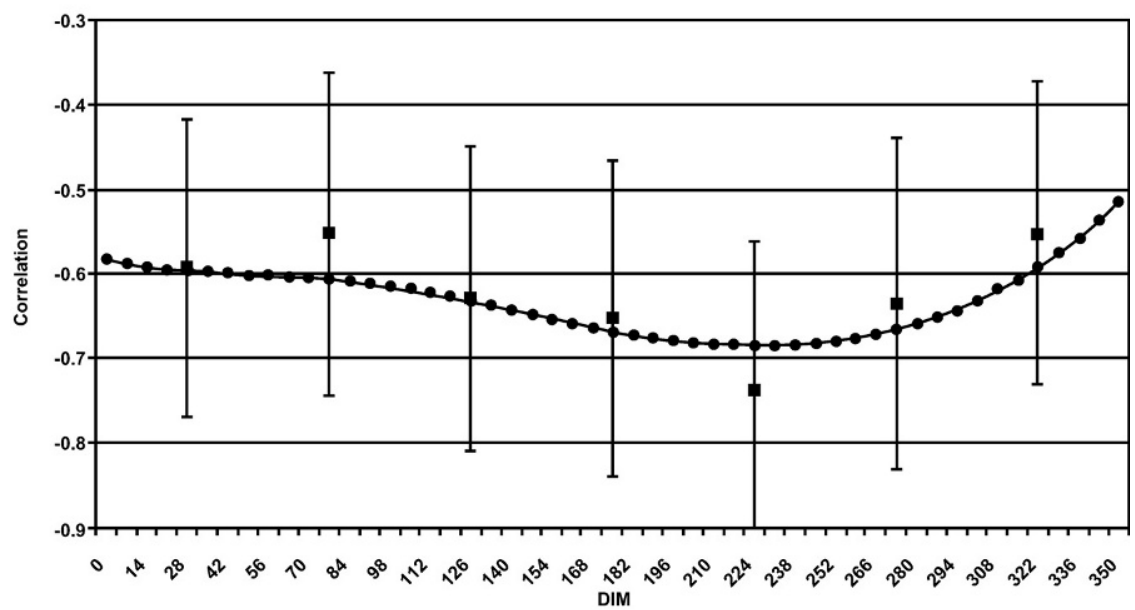

(b)

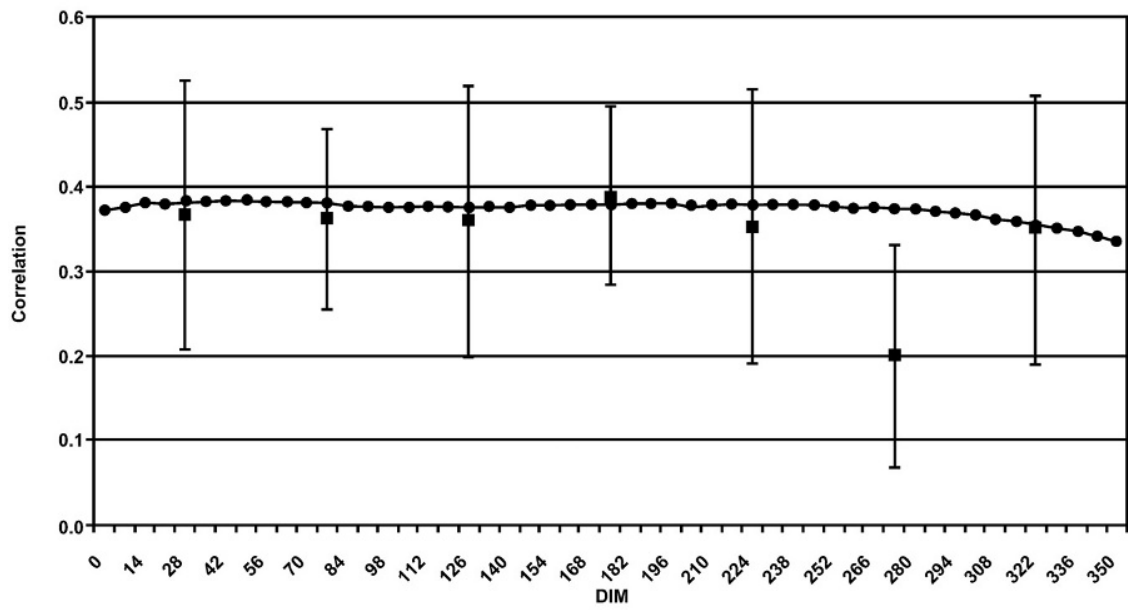

(c)

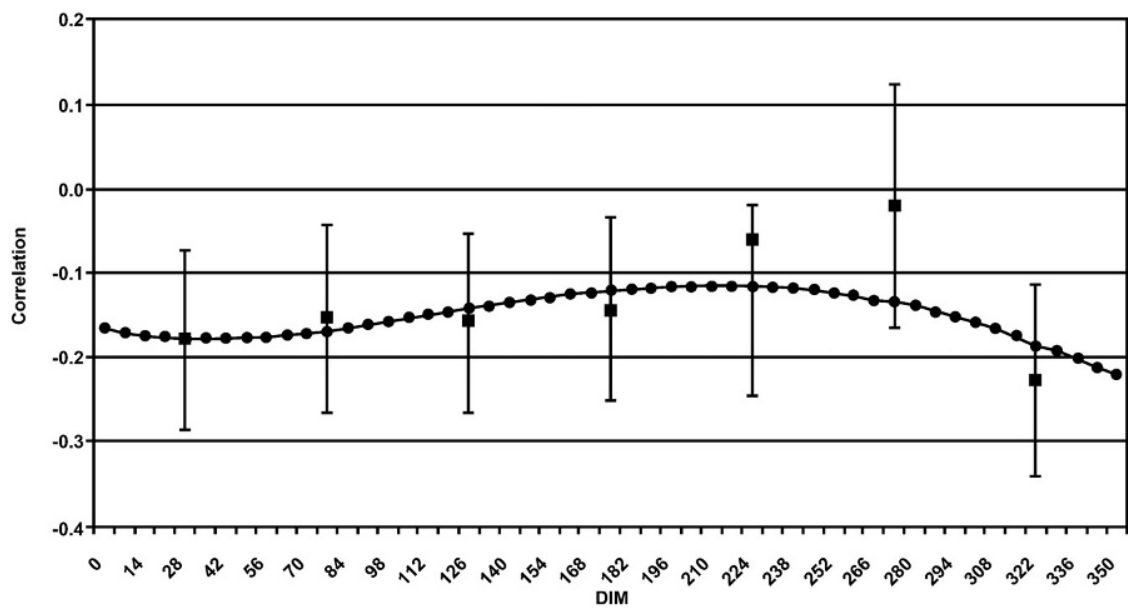

Figure 5. Genetic correlations between body weight at different stages of lactation (DIM) with (a) interval to first service ( random regression model; $\square$ multivariate); (b) number of services ( random regression model; $\square$ multivariate); and (c) pregnant 63 d after the start of breeding ( random regression model; multivariate). Also included in the graph are the SE of the correlations for the multivariate analysis. 
(a)

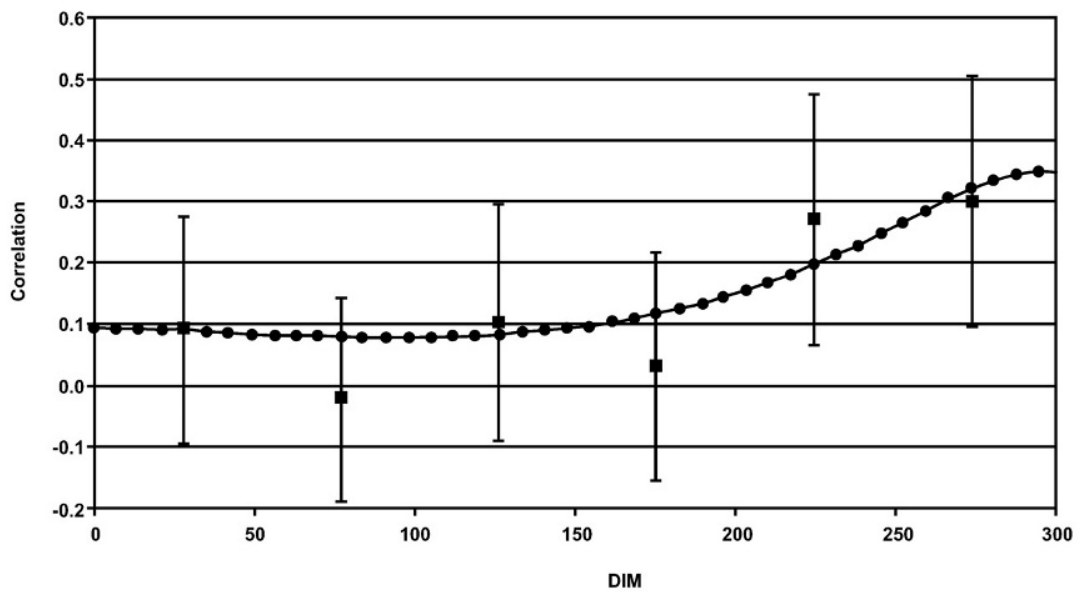

(b)

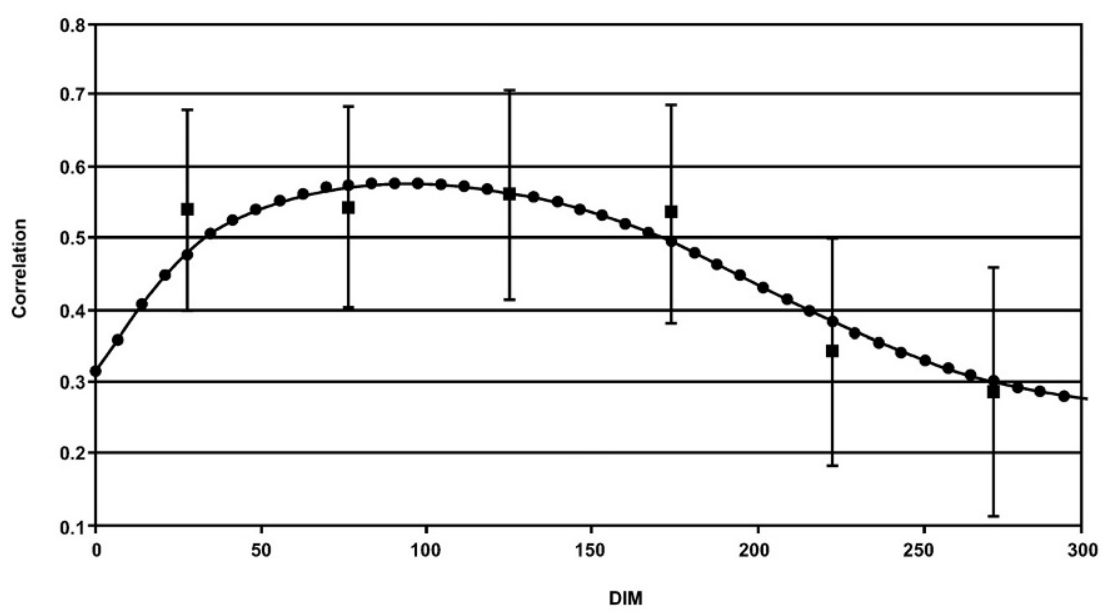

(c)

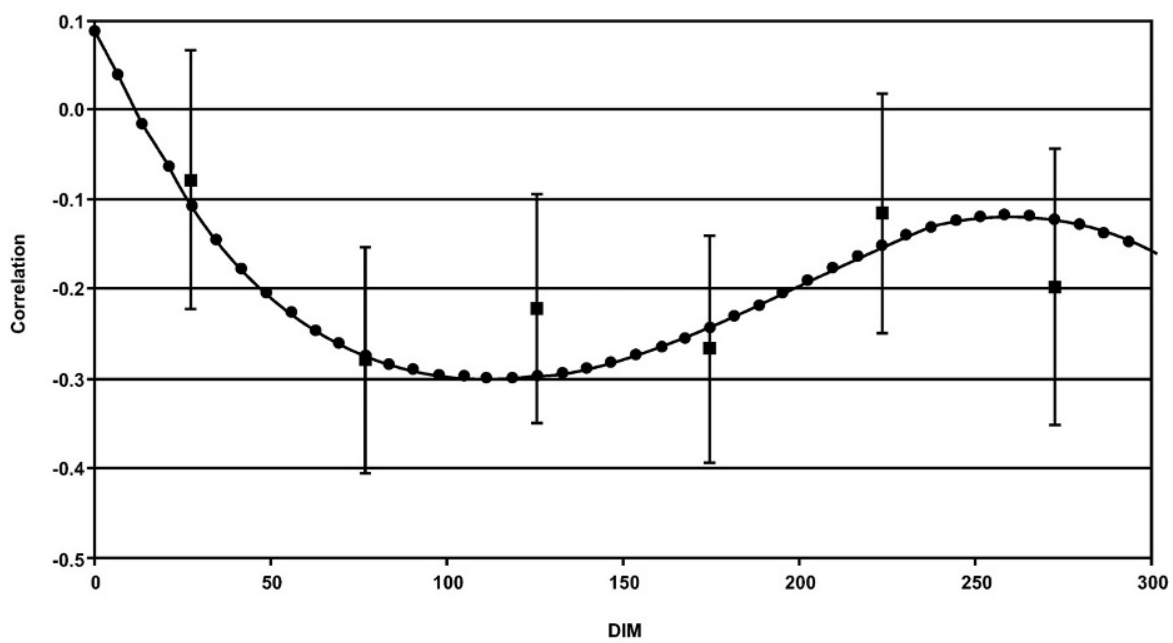

Figure 6. Genetic correlations between milk yield at different stages of lactation (DIM) with (a) interval to first service ( random regression model; multivariate); (b) number of services ( random regression model; multivariate); and (c) pregnant $63 \mathrm{~d}$ after the start of breeding ( random regression model; multivariate). Also included in the graph are the SE of the correlations for the multivariate analysis. 
calculated the eigenvalue associated with an increase in order of the random regression. In the present study other nonformal tests were also used simultaneously with the likelihood ratio test to determine the most parsimonious covariance function.

The significance of the log-likelihood ratio test depends on the amount of data available (i.e., it tells us if there is enough information in the data to fit a more sophisticated model; Pool, 2000). In the present study, a relatively large number of records were available for a large number of animals resulting in the log-likelihood of the fit significantly increasing as the order of fit increased across all three longitudinal traits (Table 2 ). Across both BCS and BW the eigenvalues of the last order of the L3 model accounted for $0.2 \%$ of the additive genetic variation in those traits; hence, there is probably little difference in the estimated breeding value of animals for BCS or BW going from an L2 to an L3. Although the last order of the L3 random regression model for milk test-day yield accounted for $8.2 \%$ of the additive genetic variation, the genetic variances and genetic correlations estimated between milk yield and fertility with the quartic random regression model were more extreme at the peripheries. Therefore, despite the significance of the quartic random regression model based on the log-likelihood ratio test, it was felt that the L3 random regression model was most appropriate for modeling milk test-day yield over a lactation.

Although more pronounced for milk yield, there was a tendency for the genetic variances estimated with the random regression model to be larger than those estimated using the multivariate model. Pool (2000) also reported lower genetic variances for milk yield estimated with a series of bivariate analyses compared with different orders of random regression models. The difference in genetic variances estimated between the models may be due to the multivariate model estimating the average covariance between the DIM within each 50-d block, which is expected to be lower than the genetic variance at any particular DIM within that block. The random regression model which included only the intercept term (LO) estimated the average genetic covariance over all DIM and therefore, yielded a lower genetic variance than most of the genetic variances estimated using the multivariate model. The unsuitability of a univariate analysis within blocks agrees with Veerkamp et al. (2001) who documented generally lower genetic variances estimated with a univariate model compared with both a multivariate and a random regression model for BCS at different stages of lactation. Veerkamp et al. (2001) reported that the difference in the estimated genetic variance between the models was more pronounced at the end of lactation, agreeing with the results from the present study.

\section{Body Condition Score}

The large increase in the genetic variance for BCS at both peripheries may be due to a combination of both a paucity of data at those periods of lactation and possibly because of the mathematical properties of a polynomial which places relatively more weight on observations at each extremity (Meyer, 1998). Also there is likely to be larger random variation around parturition because factors relating to parturition are likely to effect BCS directly postpartum. It was hoped that the inclusion of days conceived as a covariate in the model would account for most of the effect of pregnancy on BCS. Also, because the residual variation was assumed to be homogenous within each block, some of the changes in residual variation within blocks may have entered the genetic component.

Nevertheless, the range of genetic variances estimated for BCS in the present study were within the range of those estimated by Koenen and Veerkamp (1998) when both BCS measures were brought to the same scale. Similarly, the genetic variance estimated by L0 was close to previous estimates from multivariate analyses converted to the same BCS scale (Gallo et al., 2001; Pryce et al., 2001; Berry et al., 2003).

The heritability for BCS agrees with previous reports from multivariate (Veerkamp and Brotherstone, 1997; Gallo et al., 2001; Berry et al., 2003) and random regression model analyses (Koenen and Veerkamp, 1998). Because changes in a trait are the differential between two measures, variances can be calculated using the variances and covariance between the two measures. By calculating both the phenotypic and genetic variances of the change in a trait, it is possible to calculate the heritability for that trait. The heritability estimate for BCS change from d 0 to 70 was 0.19 , which is larger than the 0.09 reported by Pryce et al. (2001) over a similar time interval and also larger than that reported by Berry et al. (2002) and Dechow et al. (2002) for BCS change in early lactation. The larger heritability estimate for BCS change in the present study may be attributed to the larger genetic variances for BCS estimated with the random regression model compared with the multivariate estimates from the present study and other previous multivariate estimates for the heritability of BCS (Pryce et al., 2001; Berry et al., 2002). The phenotypic and genetic SD for BCS change between d 0 and 70 of lactation was 0.32 and 0.14 BCS units, respectively, in the present study compared with 0.34 and 0.10 BCS units, respectively, reported by Pryce et al. (2001).

The genetic correlations between BCS and fertility suggest that BCS is a moderate to strong indicator of fertility; BCS in mid to late lactation expressed the 
strongest genetic relationship with fertility. The correlations suggest that increasing BCS levels will reduce the IFS, reduce NS, and increase both PRFS and PR63. The signs of the correlations agree with previous studies that looked at the relationships between BCS and IFS (Dechow et al., 2001; Pryce et al., 2001; Veerkamp et al., 2001; Berry et al., 2003), conception rate to first service (Veerkamp et al., 2001), PRFS (Berry et al., 2003), and number of services (Berry et al., 2003). The reduced IFS in cows with higher genetic merit for BCS agrees with Royal et al. (2002), who reported significantly $(P<0.005)$ shorter intervals from parturition to commencement of luteal activity in cows with higher genetic merit for BCS. Therefore, BCS can serve as a predictor for the estimated breeding value of fertility, albeit, with an accuracy no greater than the genetic correlation between BCS with the fertility trait. The main advantage of including BCS in a selection index as a predictor of fertility arises with small progeny group sizes where the fertility data and BCS data can be simultaneously included in the selection index (Berry et al., 2003) or where the fertility data may only be available after the cow has had a subsequent calving (e.g., pregnancy related traits, calving interval).

Shorter intervals from calving to first service generally predispose animals to poorer pregnancy rates to first service; the phenotypic and genetic correlations between IFS and PRFS estimated in the present study were 0.06 (s.e. $=0.009$ ) and 0.32 (s.e. $=0.274$ ), respectively. Therefore, the economic benefits of reduced intervals to first service must be weighted against the possible reduction in pregnancy rates to first service, especially when expensive semen may be used. However, the common practice in seasonal calving herds in Ireland is to service all cows once the breeding season has begun since the risk of a reduction in pregnancy rates is expected to be more than compensated by the economic penalty of waiting for the cow's next ovulation.

The changing genetic correlations throughout lactation between BCS and fertility is in contrast to that reported by Veerkamp et al. (2001), who showed that BCS in early lactation was more strongly correlated with IFS and first-service conception rate. Similarly, Pryce et al. (2000) reported a steady decrease in their inferred genetic correlations between BCS and calving interval as the lactation progressed. However, both studies were based on primiparous cows, whereas the present study was performed on multiparous cows; Coffey et al. (2002) documented different BCS profiles and energy balance profiles for cows of different parities. Nevertheless, the differences in the strength of the genetic correlations in the present study between the minimum and maximum correlation between BCS and fertility were small $(<0.24)$, which is expected due to the strong genetic correlations between BCS at different stages of lactation. This would suggest that in order to optimize genetic response in fertility through indirect selection on BCS, progeny should be assessed for BCS in midlactation, when the genetic variance and heritability for BCS is largest.

\section{Body Weight}

The genetic variance for BW estimated with the random regression model was slightly higher than previous reports using random regression model (Koenen and Veerkamp, 1998; Veerkamp and Thompson, 1999). However, the studies of Koenen and Veerkamp (1998) and Veerkamp and Thompson (1999) were both performed on primiparous cows, whereas the present study was performed on multiparous cows that may have higher variances due to their expected higher means; Coffey et al. (2002) reported an increase in mean live weight as parity increased. Heritability estimates for BW estimated with the L3 random regression model agree with previous estimates from multivariate analyses (Svendsen et al., 1994; Veerkamp et al., 2000; Søndergaard et al., 2002) and random regression model analyses (Koenen and Veerkamp, 1998).

Genetic correlations estimated with the L3 random regression model between $\mathrm{BW}$ with fertility indicate that selection for genetically heavier animals without any consideration for other traits will result in animals having shorter intervals to first service, requiring more services, and having lower pregnancy rates $63 \mathrm{~d}$ after the start of breeding than animals selected for lighter BW. The change in the trend of the correlations between BW with IFS and the other the fertility traits may be attributed in part to the already described lower pregnancy rates with earlier service postpartum. All correlations were in agreement with the genetic correlations estimated between similar traits by Berry et al. (2003) on a similar dataset after preadjusting the data with smoothing splines. The correlations also agree with another international study; Veerkamp et al. (2000) reported negative genetic correlations between BW in early $(-0.11)$ and midlactation $(-0.54)$ with commencement of luteal activity. The trend of changing correlations over the lactation observed by Veerkamp et al. (2000) also agrees with the present study in that the genetic correlations between BW and fertility tended to be strongest in mid lactation. Similarly, Berry et al. (2003) showed that BW in midlactation was more strongly related to fertility that BW directly post calving. Nevertheless, in the present study the largest range in genetic correlations between BW at different DIM with fertility was small $(<0.17)$. 
Although 12 to $45 \%$ of the genetic variation in BW may be due to differences in BCS (Veerkamp and Brotherstone, 1997; Berry et al., 2002), the opposite signs of the genetic correlations between $\mathrm{BW}$ and BCS with most of the fertility traits indicate that other factors responsible for differences in BW (e.g., size) may also have large effects on the subsequent fertility of the animal in predominantly grass-based systems. This was investigated in the present study by adjusting BW for differences in BCS by including BCS as a covariate in the model. Correlations between adjusted BW with NS, PRFS, and PR63 became slightly stronger (average $=0.08$ ), while correlations with IFS became slightly weaker (average $=0.05$ ) following adjustment for BCS. This would indicate that independent of differences in BCS, increases in BW within the Holstein-Friesian will lead to reduced overall fertility performance if fertility is not included in the breeding objective.

\section{Milk Yield}

The range of genetic variances for milk yield estimated with the L3 random regression model are in agreement with previous literature using random regression models (Veerkamp and Thompson, 1999; Pool, 2000).

The unfavorable genetic correlations estimated between milk yield and fertility are also in agreement with most previous literature reports (Hoekstra et al., 1994; Grosshans et al., 1997; Royal et al., 2000; Pryce et al., 2001; Roxström et al., 2001; Evans et al., 2002). The signs of the correlations agree well with those estimated for a similar dataset (Berry et al., 2003), although the correlations in the present study are somewhat stronger at the same DIM. However, the slightly positive genetic correlation between milk yield in very early lactation and PR63 may be an artifact of the random regression model rather than a reflection of the true correlation present.

The genetic correlations between fertility and milk yield at different stages of lactation varied more than observed for either of the other two longitudinal traits; the genetic correlations between PRFS and milk yield at different stages of lactation varied from -0.70 to -0.24 . This may be attributed to the weak genetic correlations between milk yield at different stages of lactation estimated in the present study.

The trend of changing correlations with DIM suggests that selection for higher milk yield in early lactation will have a more pronounced deleterious effect on pregnancy rates than selection for higher milk yield in late lactation. This may be attributed to a further exacerbation of negative energy balance due to the inability of feed intake to provide the total energy re- quired for each incremental increase in milk yield (Van Arendonk et al., 1991), consequently resulting in poorer fertility. Based on the genetic parameters in the present study selection for increased milk yield at d 112 of lactation alone will reduce PRFS by 3 percentage units more than selection for increased milk yield at d 259 of lactation for each $10-\mathrm{kg}$ increase in milk test-day yield. Therefore, based on the genetic correlations with fertility reported in the present study, increases in overall lactation yield should be sought through increases in milk yield in late lactation rather than increases in milk yield in early lactation, with fertility or other related traits also included in the breeding objective.

\section{CONCLUSIONS}

It can be concluded that a cubic random regression model was most appropriate for modeling BCS, BW, and milk yield over a lactation based on the data used in the present study. Genetic variances and heritability estimates for all traits agree with most other international studies. The genetic correlations between BCS at different stages of lactation and fertility were consistently favorable, although the magnitude of the correlations changed slightly over the lactation. Hence, maximum genetic gain in fertility from indirect selection on BCS should be based on measurements taken in midlactation, when the genetic variance for BCS is largest. Increases in BW were associated with shorter IFS, increased NS, reduced PRFS, and reduced PR63. Genetic correlations involving milk yield were generally antagonistic. Genetic selection for higher lactation milk yield alone through selection on increased milk yield in early lactation is likely to have a more deleterious effect on genetic merit for fertility that selection on higher milk yield in late lactation.

\section{ACKNOWLEDGMENTS}

The authors wish to acknowledge with gratitude Allied Irish Bank, the AI managers Association, Holstein UK and the Irish Holstein-Friesian Association, Dairy Levy Farmer Funds, and EU Structural Funds (FEOGA) in financing the research program. The technical assistance of D. Cliffe, T. Condon and J. Keneally, and the guidance of Dorian Garrick in the initial stages of the study are also acknowledged.

\section{REFERENCES}

Akaike, H. 1973. Information theory and an extension of the maximum likelihood principle. Pages 267-281 in 2nd Int. Symp. Info. Theory. B. N. Petrov and F. Csaki, eds. Akademia Kaido, Budapest, Hungary.

Berry, D. P., F. Buckley, P. Dillon, R. D. Evans, M. Rath, and R. F. Veerkamp. 2002. Genetic parameters for level and change of body 
condition score and body weight in dairy cows. J. Dairy Sci. 85:2030-2039.

Berry, D. P., F. Buckley, P. Dillon, R. D. Evans, M. Rath, and R. F. Veerkamp. 2003. Genetic relationships among body condition score, body weight, milk yield and fertility in dairy cows. J. Dairy Sci. 86:2193-2204.

Butler, W. R., R. W. Everett, and C. E. Coppock. 1981. The relationships between energy balance, milk production and ovulation in postpartum Holstein cows. J. Anim. Sci. 53:742-748.

Butler, W. R., and R. D. Smith. 1989. Interrelationships between energy balance and postpartum reproductive performance in dairy cattle. J. Dairy Sci. 72:767-783.

Coffey, M. P., G. C. Emmans, and S. Brotherstone. 2001. Genetic evaluations of dairy bulls for energy balance traits using random regression. Anim. Sci. 73:29-40.

Coffey, M.P., G. Simm, and S. Brotherstone. 2002. Energy balance profiles for the first three lactations of dairy cows estimated using random regression. J. Dairy Sci. 85:2669-2678.

Crosse, S. 1986. Dairy herd management information systemDAIRYMIS II. Pages 325-361 in Moorepark 25th Anniversary Publication, Part 2: Animal Health and Machine Milking, Cork, Ireland.

Dechow, C. D., G. W. Rogers, and J. S. Clay. 2001. Heritabilities and correlations among body condition scores, production traits, and reproductive performance. J. Dairy Sci. 84:266-275.

Dechow, C. D., G. W. Rogers, and J. S. Clay. 2002. Heritability and correlations among body condition score loss, body condition score, production and reproductive performance. J. Dairy Sci. 85:3062-3070.

Evans, R. D., F. Buckley, P. Dillon, and R. F. Veerkamp. 2002. Genetic parameters for production and fertility in spring-calving Irish dairy cattle. Irish J. Agric. Food Res. 41:43-54.

Gallo, L., P. Carnier, M. Cassandro, R. Dal Zotto, and G. Bittante. 2001. Test-day genetic analysis of body condition score and heart girth in Holstein-Friesian cows. J. Dairy Sci. 84:2321-2326.

Gilmour, A. R., B. R. Cullis, S. J. Welham, and R. Thompson. 2003. ASREML Reference Manual. New South Wales Agriculture, Orange Agricultural Institute, Orange, Australia.

Grosshans, T., Z. Z. Xu, L. J. Burton, D. L. Johnson, and K. L. Macmillan. 1997. Performance and genetic parameters for fertility of seasonal dairy cows in New Zealand. Livest. Prod. Sci. 51:41-51.

Hoekstra, J., A. W. van der Lugt, J. H. J. van der Werf, and W. Ouweltjes. 1994. Genetic and phenotypic parameters for milk production and fertility in upgraded dairy cattle. Livest. Prod. Sci. 40:225-232.

Irish Cattle Breeding Statistics. 2002. Andrew Cromie, ed. Print Run Limited, Dublin, Ireland.

Jamrozik, J., and L. R. Schaeffer. 1997. Estimates of genetic parameters for a test-day model with random regressions for yield traits of first lactation Holsteins. J. Dairy Sci. 80:762-770.

Jones, H. E., I. M. S. White, and S. Brotherstone. 1999. Genetic evaluation of Holstein Friesian sires for daughter condition-score changes using a random regression model. Anim. Sci. 68:467-475.

Kirkpatrick, M., D. Lofsvold, and M. Bulmer. 1990. Analysis of the inheritance, selection and evolution of growth trajectories. Genetics 124:979-993.

Koenen, E. P. C., and R. F. Veerkamp. 1998. Genetic covariance functions for live weight, condition score, and dry-matter intake measured at different lactation stages of Holstein Friesian heifers. Livest. Prod. Sci. 57:67-77.
Meyer, K. 1998. Estimating covariance functions for longitudinal data using a random regression model. Genet. Select. Evol. 30:221240.

Meyer, K., and W. G. Hill. 1997. Estimation of genetic and phenotypic covariance functions for longitudinal or 'repeated' records by restricted maximum likelihood. Livest. Prod. Sci. 47:185-200.

Pool, M. H. 2000. Breeding value estimation based on individual testday records. Ph.D. Thesis, Wageningen University, The Netherlands.

Pryce, J. E., M. P. Coffey, and S. Brotherstone. 2000. The genetic relationship between calving interval, body condition score and linear type and management traits in registered Holsteins. J. Dairy Sci. 83:2664-2671.

Pryce, J. E., M. P. Coffey, and G. Simm. 2001. The relationship between body condition score and reproductive performance. J. Dairy Sci. 84:1508-1515.

Rekaya, R., M. J. Carabao, and M. A. Toro. 1999. Use of test day yields for the genetic evaluation of production traits in HolsteinFriesian cattle. Livest. Prod. Sci. 57:203-217.

Roxström, A., E. Strandberg, B. Berglund, U. Emanuelson, and J. Philipsson. 2001. Genetic and environmental correlations among female fertility traits and milk production in different parities of Swedish red and white dairy cattle. Acta Agric. Scand. 51:7-14.

Royal, M. D., A. O. Darwash, A. P. F. Flint, R. Webb, J. A. Woolliams, and G. E. Lamming. 2000. Declining fertility in dairy cattle: Changes in traditional and endocrine parameters of fertility. Anim. Sci. 70:487-501.

Royal, M. D., J. E. Pryce, J. A. Woolliams, and A. P. F. Flint. 2002. The genetic relationship between commencement of luteal activity and calving interval, body condition score, production, and linear type traits in Holstein-Friesian dairy cattle. J. Dairy Sci. 85:3071-3080.

Søndergaard, E., M. K. Sørensen, I. L. Mao, and J. Jensen. 2002. Genetic parameters of production, feed intake, body weight, body composition, and udder health in lactating dairy cows. Livest. Prod. Sci. 77:23-34.

Stram, D. O., and J. W. Lee. 1994. Variance components testing in the longitudinal mixed effects model. Biometrics 50:1171-1177.

Svendsen, M., P. Skipenes, and I. L. Mao. 1994. Genetic correlations in the feed conversion complex of primiparous cows at a recommended and a reduced plane of nutrition. J. Anim. Sci. 72:1441-1449.

Van Arendonk, J. A. M., G. J. Nieuwhof, H. Vos, and S. Korver. 1991. Genetic aspects of feed intake and efficiency in lactating dairy heifers. Livest. Prod. Sci. 37:37-51.

Veerkamp, R. F., and S. Brotherstone. 1997. Genetic correlations between linear type traits, food intake, live weight and condition score in Holstein Friesian dairy cattle. Anim. Sci. 64:385-392.

Veerkamp, R. F., E. P. C. Koenen, and G. De Jong. 2001. Genetic correlations among body condition score, yield, and fertility in first-parity cows estimated by random regression models. J. Dairy Sci. 84:2327-2335.

Veerkamp, R. F., J. K. Oldenbroek, H. J. Van Der Gaast, and J. H. J. Van Der Werf. 2000. Genetic correlations between days until start of luteal activity and milk yield, energy balance, and live weights. J. Dairy Sci. 83:577-583.

Veerkamp, R. F., and R. Thompson. 1999. A covariance function for feed intake, live weight, and milk yield estimated using a random regression model. J. Dairy Sci. 82:1565-1573.

Wilks, S. S. 1938. The large-sample distribution of the likelihood ratio for testing composite hypothesis. Ann. Math. Stat. 9:60-62. 